\title{
SCI Papers Published by the Academies of Agricultural Sciences of Six Provinces in China and Contrastive Analysis on Research Papers
}

\author{
Zhao Jingjuan, Zheng Huaiguo, Tan Cuiping \\ Institute of Agricultural Sci-Tech Information \\ Beijing Academy of agricultural and Forestry Sciences, Beijing 100097, China
}

\begin{abstract}
Based on perspective of research papers, to evaluate the scientific research situation, levels, and perfoemance of six Academy of agricultural science objectively, and provide references for scientific research management and discipline construction. In this study, the papers published by authors of Beijing Acad Agr \& Forestry Sci, Shandong Acad Agr Sci Zhejiang Acad Agr Sci, Jiangsu Acad Agr Sci, Guangdong Acad Agr Sci, and Shanghai Acad Agr Sci were retrieved from SCI databases. These papers were subjected to objective comparison and evaluation of research performance among Academy of agricultural science using the indicators of the total quantity and growth of papers, citations per document, and high quality papers. A comparsion of different disciplines was also investigated. The results shown: The six provinces of academic papers showed rapid growth, with the quantity of output growth, influence gradually improve, subject distribution widely, institutions subject prominent advantage.
\end{abstract}

KEYWORD: Research institutions; SCI; Academic papers; Bibliometrics

\section{INTRODUCTION}

Scientific and rational research evaluation is significantly important to the promotion of the rational allocation and flow direction of the limited scientific and technological resources and the guaranteeing of the advancement of the country's decision-making system. Agriculture institutions are the important base for national agricultural science and technology innovation, and to improve the management over scientific researches and conduct real-time research evaluation is very meaningful to the improvement of the scientific and technological level of the agriculture institution.

As one of the major scientific achievements, scientific paper has become an important indicator of technology evaluation and measurement. Evaluation and analysis of the output and impact of researches has become a common practice at home and abroad for performing scientific research evaluation. From the perspective of academic papers, this paper focuses on Beijing Academy of Agriculture and Forestry Science, Shandong Academy of Agricultural Science, Zhejiang Academy of Agricultural Science, Jiangsu Academy of Agricultural Science, Guangdong Academy of Agricultural Science and Shanghai Academy of Agricultural Science to conduct contrastive study from the aspects of bibliometric indicators as research paper number and frequency of citations, the growth trend of papers, highly influential papers and discipline distribution, so that the research status, levels and achievements of these scientific agricultural research institutions can be objectively evaluated, and a reference for research management and discipline construction can be provided.

\section{SELECTION OF DATABASE}

SCI data base is taken as the data source of this study. The source journals of the SCI data have gone through strict quality selection and have advantages in reference data. SIC data base is not merely an important search tool, but also a significant basis for the evaluation of scientific research findings. A lot of countries in the world take it as an official or unofficial evaluating tool. Therefore, data acquired based on SCI-oriented statistics and analysis is authentic and representative.

\section{DATA COLLECTION AND PROCESSING}

The major English expressions of the research institutions and their subordinate research organizations are listed according to their official websites. Based on it, key words are extracted, search fields are added and small-scale pilot searches are conducted; the perfect search strategies 
are established after cleaning and screening.

By cleaning the data with TDA software, this paper manages to find the data of papers published by the first authors and corresponding authors of each research institution. In this study, the retrieval data is December 20th, 2013; the paper publication year is 2003-2012; the document types are Article and Review. After the above cleaning, 1,247 data from 6 institutions are acquired.

Excell is used as the data analytical tool. The research contents include the comparison of number of papers, paper growth, paper influence and discipline distribution of Beijing Academy of Agriculture and Forestry Science and other five academies of agricultural science.

\section{NUMBER OF SCI ACADEMIC PAPERS AND GROWTH TREND}

In order, the numbers of autonomously published SCI papers by the six academies of agricultural science from 2003-2012 are: Beijing Academy of Agriculture and Forestry Science, 274; Shandong Academy of Agricultural Science, 268; Zhejiang Academy of Agricultural Science, 264; Jiangsu Academy of Agricultural Science, 145; Guangdong Academy of Agricultural Science, 120; Shanghai Academy of Agricultural Science, 176. As for the total numbers of paper published by the first authors or corresponding authors, Beijing Academy of Agriculture and Forestry Science ranked the first; Shandong Academy of Agricultural Science took the second place with 268 papers; Zhejiang Academy of Agricultural Science ranked third place with 264 papers.

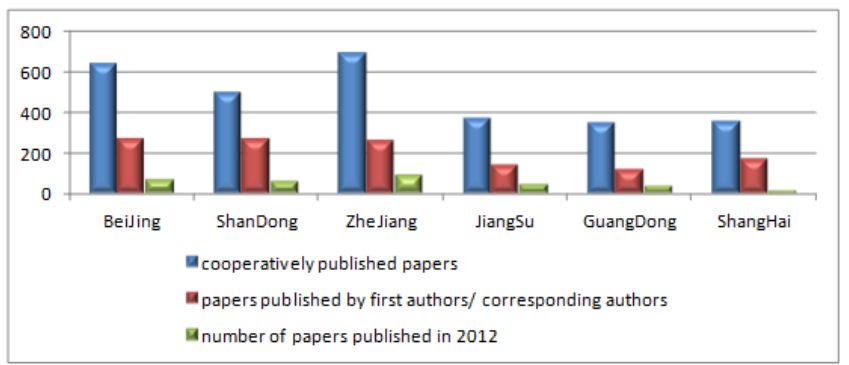

Figure. 1 Comparison of number of SCI academic papers published by the six academies of agricultural science

It can be seen from Figure 3 that, before 2006, without expectation, the publication of SCI papers of the six academies of agricultural science was at the initial stage. Beijing Academy of Agriculture and Forestry Science and Shanghai and Zhejiang Academy of Agricultural Science published more papers while Shandong and Guangdong Academy of Agricultural Science published fewer. In general, nevertheless, the difference was not significant; 2007 was the start point of rapid development. Beijing Academy of Agriculture and Forestry
Science and Zhejiang Academy of Agricultural Science exceeded the other four academies of agricultural science with strong growth and managed to maintain the stable growth trend in the following several years. In contrast to the other five academies of agricultural science, Beijing Academy of Agriculture and Forestry Science ranked second place in the number of cooperatively published papers from 2003-2012, second only to Zhejiang Academy of Agricultural Science; and the number of its autonomously published papers ranked first place, followed by Shandong Academy of Agricultural Science in the second and Zhejiang Academy of Agricultural Science in the third place.

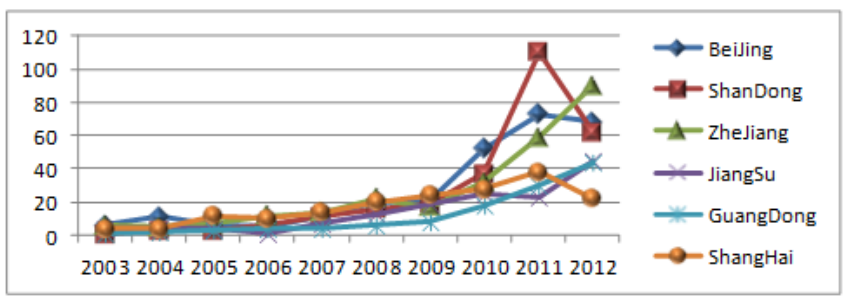

Figure. 2 Publication trend of SCI papers of the six academies of agricultural science in the ten years (published by the first author or corresponding author)

\section{CONTRASTIVE ANALYSIS OF THE IMPACT OF SCI ACADEMIC PAPERS}

Paper production is an important indicator that reflects the number of national or institutional papers, while citation frequency is an important indicator that reflects paper quality. Citation frequency of scientific paper, a basic indicator for evaluating the institutional scientific level and paper influence from the angle of users, reflects the quality of scientific papers published by the institution. The citation frequency of the autonomously published papers published by Beijing Academy of Agriculture and Forestry Science was 972 from 2003-2012, and the average citations per paper were 3.55 .

Table 1 Comparison table of influence of SCI papers published by the six academies of agricultural science

\begin{tabular}{|c|c|c|c|c|c|c|}
\hline & Beijing & Shandong & Zhejiang & Jiangsu & Guangdong & Shanghai \\
\hline Total amount of citations of the 10 years & 972 & 1180 & 1121 & 550 & 601 & 1627 \\
\hline $\mathrm{TC} \geqq 30$ published papers & 4 & 6 & 1 & 1 & 5 & 12 \\
\hline $\mathrm{TC} \cong 20$ published papers & 10 & 9 & 8 & 4 & 7 & 24 \\
\hline $\mathrm{TC} \geqq 10$ published papers & 24 & 39 & 38 & 16 & 18 & 51 \\
\hline $0<\mathrm{TC}<10$ published papers & 145 & 149 & 172 & 83 & 60 & 90 \\
\hline $\mathrm{TC}=0$ publication & 105 & 80 & 64 & 51 & 42 & 36 \\
\hline $\mathrm{TC}=$ percentage of 0 publication & $38.3 \%$ & $29.9 \%$ & $24.2 \%$ & $35.2 \%$ & $35 \%$ & $20.5 \%$ \\
\hline Average citations per paper & & 4.4 & 4.2 & 3.8 & 5.0 & 9.2 \\
\hline Percentage of highly influential papers & $15.1 \%$ & $17 \%$ & $17 \%$ & $7.5 \%$ & $9.4 \%$ & $34 \%$ \\
\hline Maximum citations frequency per paper & 40 & 73 & 70 & 35 & 61 & 91 \\
\hline
\end{tabular}

In the aspect of paper influence, Shanghai Academy of Agricultural Science, with the largest number of papers with citations frequency $\geqq 20$ and $\geqq 30$, ranked first place in total citations and average citations per paper, indicating that although with a slightly smaller number of paper than Beijing academy of Agriculture and Forestry Science and Zhejiang Academy of Agricultural Science, 
Shanghai Academy of Agricultural Science had significant advantages in paper quality and influence. Despite the fact that Beijing Academy of Agriculture and Forestry Science performed better in number of SIC papers, in paper influence, it ranked fifth place both in total citations and average citations per paper. The paper quality still needed to be promoted.
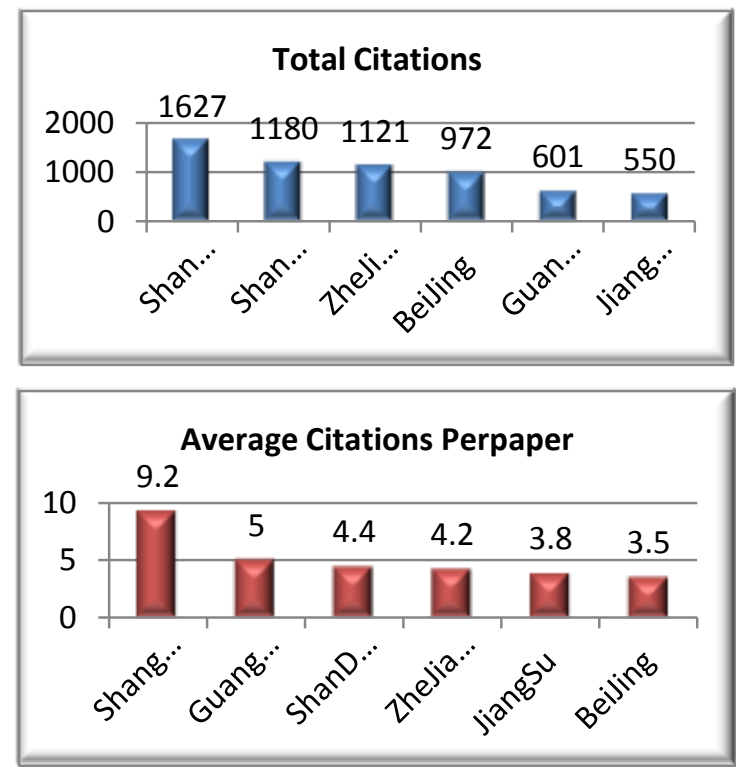

Figure. 3 Comparison chart of total citations and average citations per paper of SCI papers published by the six academies of agricultural science from 2003-2012

Sort the 1,263 SCI papers published by the six academies of agricultural science from highest citations to lowest and select the top 100 papers. 106 highly influential papers are got, accounting for $8.4 \%$ of all the SCI papers. The total citations of the 106 papers were 2,838 and the average citations per paper were $26.8,5.6$ times greater than the average citations per paper of all the 1,263 papers.

Of all these 106 highly influential papers, the maximum citation frequency was 91 , and the minimum was 14 . The citations showed an obvious long-tail skewed distribution. 8 papers, accounting for $7.5 \%$ of all the highly influential papers, had been cited for more than 50 times; 44 papers, accounting for up to $41 \%$ of all the highly influential papers, had been cited for less than 20 times.

Of all the highly influential papers, the percentage accounted by Shanghai Academy of Agricultural Science was the highest. It had 36 such papers, accounting for $34 \%$ of all the highly influential papers; Zhejiang and Shandong Academy of Agricultural Science followed Shanghai Academy of Agricultural Science with 18 highly influential papers accounting for $17 \%$ respectively; Beijing Academy of Agriculture and Forestry Science ranked fourth place with 16 highly influential papers, accounting for $15.1 \%$; Guangdong Academy of Agricultural Science ranked fifth place with 10 highly influential papers, accounting for 9.4\%;
Jiangsu Academy of Agricultural Science ranked sixth place with 8 highly influential papers, accounting for $7.5 \%$.

\section{CONTRASTIVE ANALYSIS OF THE DISCIPLINE OF SCI ACADEMIC PAPERS}

Since SCI discipline classification is based on the journals and not suitable for specific academic discipline analysis, this study paper conducts reclassification and re-indexing paper by paper for all the documents of the six academies of agricultural science and makes statistics in accordance with the classification results, as shown in Figure 5. The SCI scientific papers published by the six academies of agricultural science mainly focus on basic agricultural science, crop, plant protection, agricultural information science, animal science and other major disciplines.

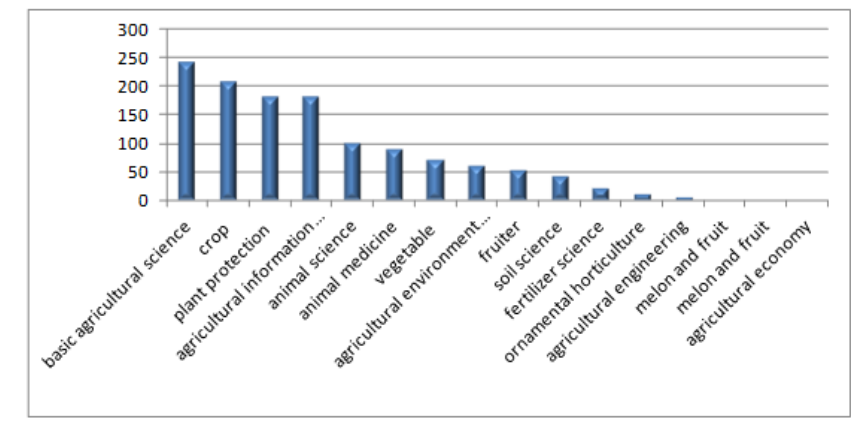

Figure. 4 Discipline distributions of SCI scientific papers published by the six academies of agricultural science

The contrastive analysis on the number of SCI papers published based on academic disciplines shows that, the preponderant disciplines of Beijing Academy of Agriculture and Forestry Science were agricultural information science, forestry, agricultural engineering, melon and fruit, fruiter and vegetables, etc, among which the most outstanding discipline was agricultural information science. The number of SCI scientific papers of this field accounted for $40 \%$ of all the SCI scientific papers of the academy, and the document amount even exceeded the total amount of the SCI papers published by the other five academies of agricultural science of this field. The preponderant disciplines of Shandong Academy of Agricultural Science were basic agricultural science, crop, animal science, animal medicine, etc; the preponderant disciplines of Zhejiang Academy of Agricultural Science were plant protection and vegetable; the outstanding disciplines of Jiangsu Academy of Agricultural Science were ornamental horticulture and agricultural environment sciences; Guangdong Academy of Agricultural Science had published fewer SCI papers, but it had published the largest number of papers on animal science research and also had published a large number of papers on 
fruiter research; the preponderant discipline of Shanghai Academy of Agricultural Science is vegetable research, and the number of papers published by it in this field equaled to the paper number of Beijing Academy of Agriculture and Forestry Science and Zhejiang Academy of Agricultural Science.

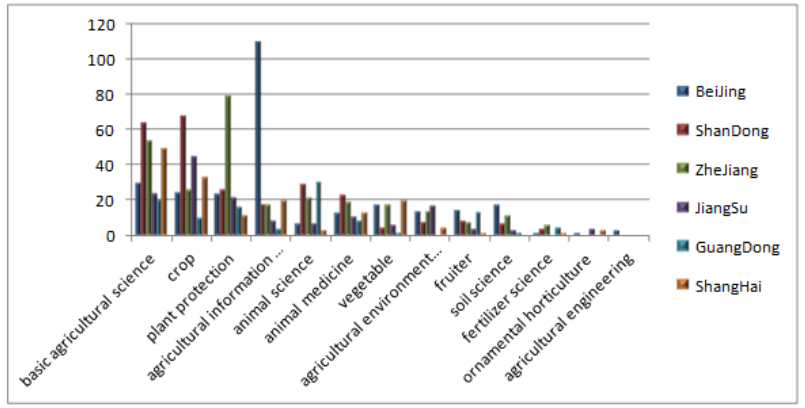

Figure. 5 Detailed distribution of SCI scientific papers published by the six academies of agricultural science

\section{CONCLUSION}

Based on the data source of SCI data base and the application of bibliometric methods, this paper focuses on Beijing Academy of Agriculture and Forestry Science and other Chinese academies of agricultural sciences in six provinces and cities and conducts comprehensive and objective contrastive study from the aspects of paper number, paper growth trend, frequency of citations, number of highly influential papers and discipline distribution. Based on the analysis and summary, the following conclusions can be drawn:

(1) Beijing Academy of Agriculture and Forestry Science ranked first with 274 autonomously published SCI papers from 2003-2012 and maintained a rapid growth in the past five years. Shandong Academy of Agricultural Science and Zhejiang Academy of Agricultural Science ranked the second and third place, respectively.

(2) In the ten years, Shanghai Academy of Agricultural Science ranked the first in total citations, average citations per paper and the number of highly influential papers. Thus it can be seen that, although Shanghai Academy of Agricultural Science had a smaller number of published papers than Beijing Academy of Agriculture and Forestry Science and Zhejiang Academy of Agricultural Science, it has apparent advantages in paper quality and influence.

(3) It can be seen from the perspective of the discipline analysis of SCI academic papers that, the preponderant disciplines of Beijing Academy of Agriculture and Forestry Science are agricultural information science, soil science, forestry, agricultural engineering, melon and fruit, fruiter and vegetable, etc., and the most outstanding discipline is agricultural information science; the preponderant disciplines of Shandong Academy of Agricultural Science are basic agricultural science, crop, animal science, animal medicine, etc; the preponderant disciplines of Zhejiang Academy of Agricultural Science are plant protection and vegetable; the outstanding disciplines of Jiangsu Academy of Agricultural Science are ornamental horticulture and agricultural environment science; Guangdong Academy of Agricultural Science had published fewer SCI papers, but it had published the largest number of papers on animal science research and also had published a large number of papers on fruiter research; the preponderant discipline of Shanghai Academy of Agricultural Science is vegetable research, and the number of papers published by it in this field equaled to the paper number of Beijing Academy of Agriculture and Forestry Science and Zhejiang Academy of Agricultural Science. The discipline distribution of papers published these academies of agricultural science are closely connected to the regional environment and local features and have given prominence to the local features.

\section{REFERENCES}

[1] H.J. SUN, Q. ZHOU, S.J. CHEN, Study on Scientific research performance of six domestic and foreign agriculture-related universities: Based on perspective comparison of Wos papers, Journal of China Agricultural University, Vol. 1(2014), p. 231-240.

[2] J.B. GU, Y.D. CHEN, L. LIANG, Research on the Evaluation Indicators of Strength and Quality of the National Science\& Technology Based on the Output of Research Papers, R\&D Management, Vol, 2(2008), p.107113.

[3] J.G. LIU, The Appraisal of the Academic Influence of Universities' Library in Tianjin on the Basis of CNKI, Library Tribune, Vol. 4(2010), p. 27-30.

[4] J.W. JU, H.Q. ZHAO, Analysis of SCI Papers of Major Agricultural Universities in Chinese Mainland Based pm SCI-E, Journal of Library and Information Sciences in Agriculture, Vol. 2(2011), p. 16-19.

[5] L.W. SHI, A Discipline strength analysis between the 4Chinese agricultural universities and the 5agriculturerelated universities from Europe and America, Journal of China Agricultural University. Vol. 3(2012), p. 192-198.

[6] Y.Q. XU, Z.P. AN, W.H.YAO, A Comparative study of henan's universities producing cssci theses and their academic influence, Journal of Henan University of Technology( Social Science), Vol. 4(2009), p. 72-75.

[7] Y.Q. WANG, Statistical Analysis of Publications of Chinese Academy of Agricultural Sciences Based on SCIE, Journal of Library and Information Sciences in Agriculture, Vol. 8(2012),p, 54-59.

[8] Y.M. Li, An Analysis of Subject Development and Research Performance on Web of Science-A Case Study of Hubei University for Nationalities, Journal of Modern Information, Vol. 9(2012), p. 97-101.

[9] T.WANG, Y. YAN, Y.H. XU, Analysis of Output and International Academic Influence of Academic Papers of Chinese Academy of Agricultural Sciences - Based on Web of Science Database, Journal of Agricultural Science and Technology, 2013, Vol. 15(2), p. 54-63. 